\title{
Small shortwave whip antenna broadband research
}

\author{
Li Yin ${ }^{1,}$ a Zhi-Gang Zhang, ${ }^{2, b}$ and Jin-Peng Zhu ${ }^{3, c}$ \\ ${ }^{1}$ College of Electronic Engineering, Naval University of Engineering, \\ Wuhan 430033, China \\ ${ }^{2}$ College of Electronic Engineering, Naval University of Engineering, \\ Wuhan 430033, China \\ ${ }^{3}$ Institute of China electronics technology group co.LTD.22, Qingdao \\ 266071, China \\ ahjgyinli@163.com, ${ }^{b}$ submarinezzg@163.com, ${ }^{c}$ liting89720@126.com
}

\begin{abstract}
It is designed a pair of $5 \mathrm{~m}$ whip antenna for antenna arrays, the integrated use of a centralized method of loading and top loading, which is reducing the sensitivity of the antenna impedance versus frequency and using the moment method and genetic algorithm to load the network number so that the location load resistance element can be to optimize the design, and the actual production of the antenna test results are consistent with the simulation results, and the test results show that the antenna VSWR within $5 \sim 20 \mathrm{MHz}$ frequency range of less than 2.5.
\end{abstract}

Keywords: Whip antenna, antenna, broadband, shortwave

\section{Introduction}

The engineering requirements and design of an antenna array system that requires unit antennas on the following main points:

(1)In the operating frequency band $(5 \sim 20 \mathrm{MHz})$, the direction of the antenna pattern remains substantially stable;

(2)Try to simplify the antenna matching network to ensure the amplitude and phase of each antenna element that is consistent;

(3) Receiving antenna to have a certain efficiency;

(4)The antenna structure is simple, lightweight, fast handling, reliable, durable, height does not exceed $5 \mathrm{~m}$.

It is commonly used in shortwave band broadband antenna and has a conical cage antenna, but the two larger antenna profiles; the sleeve antenna itself has a wider characteristic, and the antenna electrical characteristics of the tapered is less different and in low profile, and the receive array can be used as the basic unit, but the bandwidth of the antenna can be typically 2 to 3 octaves, also slightly heavy 
volume antenna; whip antenna has an omni directional horizontal pattern, a simple structure, and the outline of the characteristics of low production cost is also low, which often is used as the antenna element for antenna array, this paper in order to simplify the antenna units, the height of antenna is adopted using $5 \mathrm{~m}$ of the whip antenna type. In the main antenna band $5 \sim 20 \mathrm{MHz}$ band, electric height $5 \mathrm{~m}$ antenna at low frequencies are less than 0.1 wavelength, which are electrically small antenna, this time with a real part of the input impedance of small, large capacitance, and the resistance is characterized by rapid change, we must take measures to reduce the sensitivity of the antenna input impedance versus frequency.

\section{Concentrated load design}

The whip antenna VSWR has been achieved with a more effective way that is the broadband antenna body to be loaded ${ }^{[1]}$. Generally, the loading may be a passive device, which can be active network. For the introduction of active networks, even though it can achieve miniaturization of antennas, broadband segment, easy electronic control, etc., but it would inevitably lead to an active device noise, nonlinear distortion, amplitude and phase consistency for demanding phased array antennas is not suitable, therefore, the design uses a passive load.

Common passive loading methods: top-loading, medium load, load distribution, the lumped element loading and so on. In the shortwave band, medium load and load distribution is difficult to achieve, it is more convenient to use top-loading and loading lumped element. Lumped element resistive element can also be used to load reactance element: reactance loading, high antenna efficiency, but the band is narrow, it is difficult to meet within four octave broadband requirements; and resistive loading, although the antenna bandwidth, but low efficiency; reactance and resistance of hybrid network load, ease the contradiction between the antenna band and efficiency. Loading of the antenna design is to optimize the position and number of the loaded component values and loading points, the goal of optimizing the efficiency and the high input impedance of the planar antenna, but generally, the efficiency and the impedance matching between the two is a conflict, which needs strike a balance between the $\operatorname{two}^{[2]}$, we use the method of moments on the antenna performance computing.

In the method of moments, the time zone using the excitation voltage source, the applied electric field can be expressed as:

$$
E_{i z}=V \delta(z)
$$

In it, $V$ is excitation voltage.

For concentrated load, the load area length is smaller than the length of the antenna cases, which can be used instead of the Dirac function and distribution of the loading area in the impedance domain

$$
Z(z)=Z_{i} \delta\left(z-Z_{i}\right)
$$


In the formula: $Z_{i}$ is the lumped load element impedance; ${ }^{Z_{i}}$ is the center position for the loading area

$$
\int_{-L}^{L} I\left(z^{\prime}\right) g(r) d z^{\prime}+\frac{1}{j \eta_{0}} \int_{0}^{z} Z\left(z^{\prime}\right) I\left(z^{\prime}\right) \sin k_{0}\left(z-z^{\prime}\right) d z^{\prime}=C \cos k_{0} Z+\frac{V}{j 2 \eta_{0}} \sin k_{0}|z|
$$

(3)

In the formula: $\eta_{0}=\sqrt{\mu_{0} / \varepsilon_{0}}$ wave impedance of free space, $k_{0}=\omega \sqrt{\mu_{0} \varepsilon_{0}}$, $C$ for integration constant.

Moment method to calculate the antenna current distribution can be obtained and then get the other parameters of the antenna radiation

Since the single whip antenna input impedance is small at low frequencies resistance portion, resulting in matching difficulty, so it is considered the root of the antenna loading, single resistor load for a great impact on the efficiency of the antenna. Therefore, considering the parallel load, wherein $L=6 \mu \mathrm{H}, C=300 \mathrm{pF}$, after the use of different resistance loads, the VSWR and radiation efficiency of the antenna shown in Figure 1
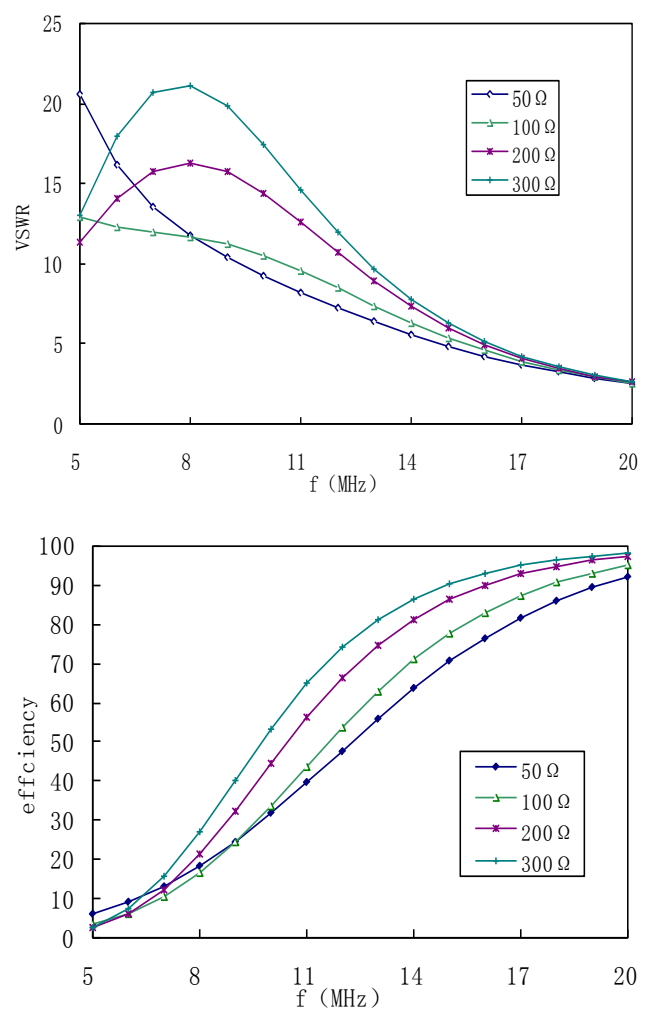
Figure 1. Bottom loading, the VSWR and radiation efficiency of different resistance

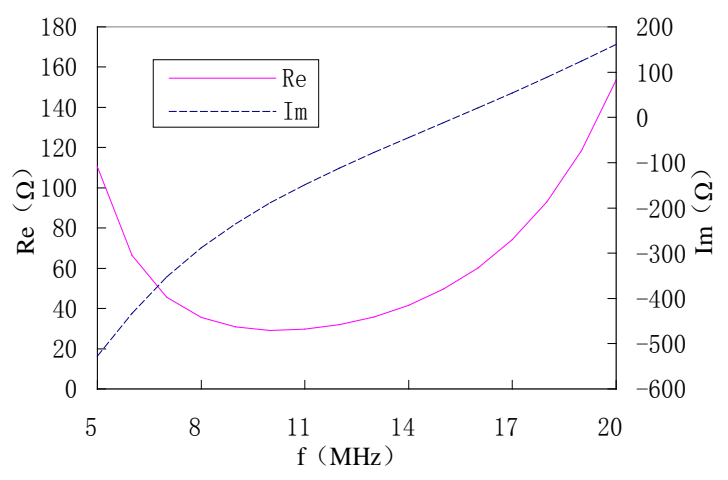

Figure 2. Bottom loading 200 ohm

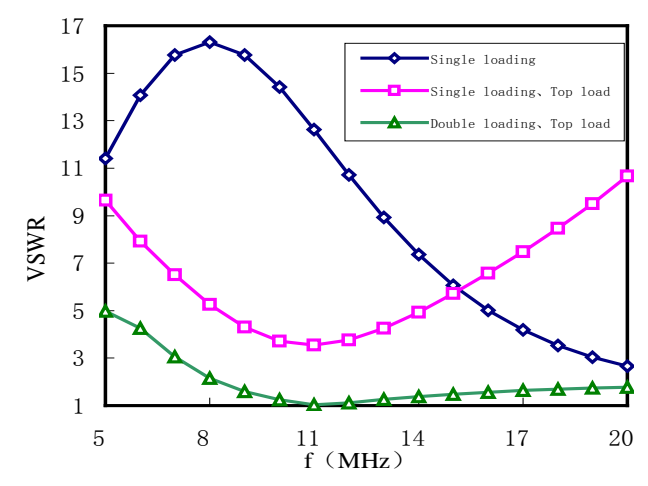

Figure 3. Using different loading input impedance of the antenna schemes antenna VSWR

From the simulation results, when using the bottom of a single load, it has impact on the load resistance and impedance characteristics of the antenna on the efficiency, if the load resistance is small, the overall of radiation efficiency of the antenna will be low, and from the integrated VSWR and efficiency point of view, it will be appropriate to select $200 \mathrm{ohm}$ of the bottom of the load. Compared with the before and after loading of the antenna VSWR, it can been seen that a single load can be increased significantly to reduce low frequency of antenna VSWR, which played a role in smoothing the input impedance of the antenna, but the VSWR is still high, As can be seen from Figure 2, bottom load effectively increases the input resistance of the low frequency, but the impact on the reactance 
part is small, the overall capacitive reactance value is high, it is also necessary to further reduce the sensitive input impedance versus frequency degree.

\section{Top load design}

At the top of the whip antenna is loaded with pellets, discs or radiation lobe which is called a top load, and the top load is increased due to the top of the vertical portion of the distributed capacitance to ground but it is no longer open, however, the current is not zero and the effect is equivalent to a capacitor that has been loaded at the top, without increasing the effective height of the antenna under the improved geometry of the height of the antenna radiation resistance that is increased, while reducing the input capacitance of the antenna, to effectively improve the performance of the antenna matching in the low frequency band.

The reference ${ }^{[3]}$ showed that the two loading points can make the input impedance of the antenna whip $7 \mathrm{~m}$ within $3 \sim 30 \mathrm{MHz}$ frequency with smooth range greatly, usually on the loading point for the RL parallel loading, and the loading point is for RLC parallel load. For on a height of $5 \mathrm{~m}$, the diameter is not exceeding $50 \mathrm{~mm}$ single whip to achieve the VSWR and efficiency (gain) broadband ( $5 \sim 20 \mathrm{MHz}$ ), the design of an integrated centralized loading and top loading programs, and the use of numerical algorithms of the antenna loading position, the loading element values were preliminary an optimized design, and a single load, single top load, dual antenna VSWR load top load programs were shown in Figure 3. As it can be seen from the figure, the lumped element loading and top loading can effectively smooth the input impedance of the antenna to improve the matching performance of the antenna, the use of dual loading top loading regimen, in addition to the standing wave ratio of the antenna outside the low-frequency side, which are designed to meet the requirements, taking into account the resistance of the low frequency band and is mainly small, great electrical resistance, a matching circuit may therefore be solved.

\section{Antenna Matching Network design}

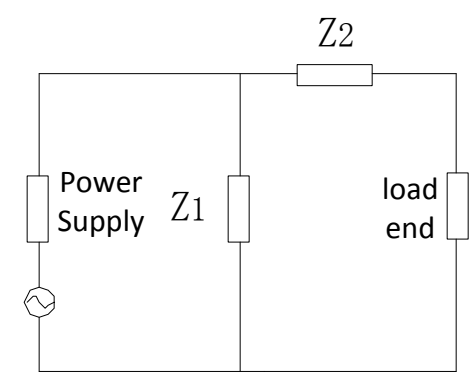

Figure 4. $\Gamma$ shape matching network

While the loading of the antenna body greatly smoothes the input impedance of the antenna, but in a transverse dimension it is smaller than the wavelength of 
the vertical antenna feed line to achieve matching with the fixed impedance of the antenna that is also required between the antenna and the receiver plus the matching network ${ }^{[4]}$. For the antenna array, the need for precise control of each element antenna array amplitude correlation coefficient to achieve spatial filtering, so in order to increase the amplitude and phase coherence unit antenna in the design process, it is tried to simplify the antenna matching circuit, reducing the nonlinear device and the phase instability.

Antenna system commonly used in the matching network is lumped network, in order to improve the radiation efficiency of the antenna is mainly passive, by reactance element ( $L, C$ ) with $T$ form, $\Gamma$ form, and $\Pi$ form in the group of the networks ${ }^{[5]}$, a common kind of impedance matching network is a pure reactance-shaped network structure shown in Figure 4. Usually it is close to purely resistive impedance of the power supply side, in order to better match the supply side, the requirements reactance $\mathrm{Z} 1, \mathrm{Z} 2$ needs the contrary nature. For passive matching network, usually it is inserted to represent the attenuation and phase shift of a network system applied to the circuit that affect the outcome of the load, when the inductive reactance $\mathrm{Z} 2, \mathrm{Z} 1$ is the capacitive reactance to form a parallel resonant circuit, which will cause the phase lag of the network. When Z2 is capacitive and Z1 is the inductance, they will constitute a series resonant circuit, caused by a phase advance network. It can select the appropriate form of network according to the actual impedance characteristics of the antenna to achieve optimum impedance matching.

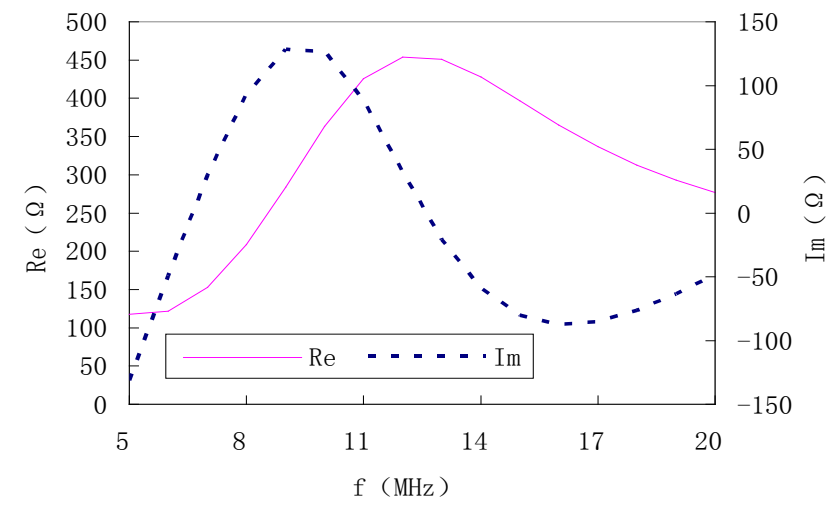

Figure 5. The input impedance matching circuit of $5 \mathrm{~m}$ of the whip antenna after it has been increased

It can be seen from Figure 5, after the use of the matching circuit, the input impedance of the real part of the antenna is between 120 to 450 ohms, and between the imaginary part of -130 to 130 ohms up and down, which directly connected to the $50 \mathrm{ohm}$ impedance of the receiver due to the over-large difference to adapt, and it is also necessary to increase a broadband transmission line of transformer to achieve the impedance transformation ratio. 
The impedance transforming characteristics of the transmission line transformer is very complicated, which is not a fixed ratio of $4: 1$ and it will vary with the change in frequency and load impedance. By adjusting the various parameters of the transmission line transformer and the antenna body, the variation of the transmission line transformer turns ratio and variation of the antenna input impedance, which is preferably coordinated, and the degree of fluctuation of the transformed impedance will be greatly reduced, thereby obtaining a broadband match.

The optimized loading of the antenna of the final design indicators are as follows: the load pitch to the top of the antenna is $1 \mathrm{~m}$, and the element value is: $R_{1}=300 \Omega, L_{1}=6 \mu \mathrm{H}$. The below loading point of the antenna element value is: $R_{2}=250 \Omega, L_{2}=6 \mu \mathrm{H}, C_{2}=300 \mathrm{pF}$, and the top of the radiation load using the 4 leaves program, $1.5 \mathrm{~m}$ of the length, the inclination angle of 45 degrees, the resulting standing wave antenna is as shown in Fig.

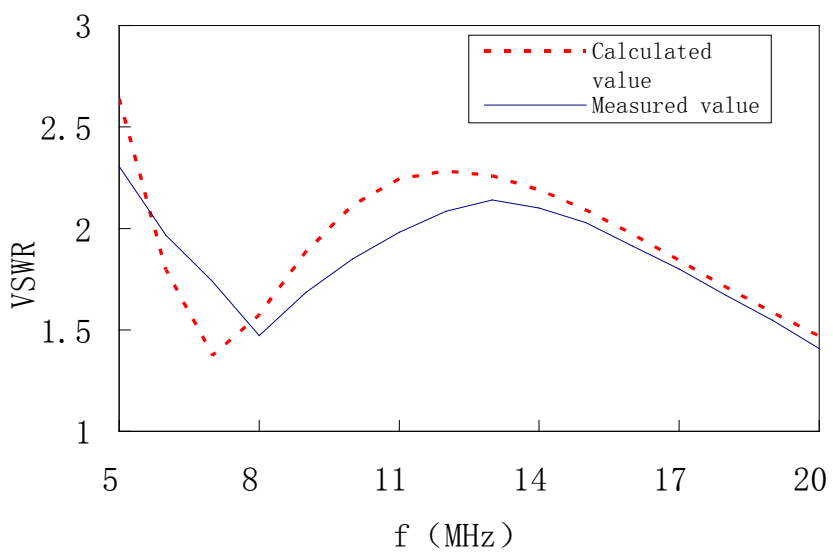

Figure 6. The VSWR of the antenna after optimized design

The trend of the tested and calculated values are basically the same, in general, the actual measured value is slightly lower than the calculated value, and the difference is mainly influenced by the following factors: 1) the matcher is for nonlinear element, and the VSWR role is by certain optimization; 2) The actual test field and the ideal of the calculation has a certain difference such as in the conductivity of ground, and the influence of the surrounding objects, etc. Test results show that both the antenna VSWR of 2.5 or less, reached the indicator of the design.

\section{Conclusion}

This paper presents five meters short-wave whip antenna pair for the antenna array, the integrated use of a centralized top-loading method and the method used to load the operating band of the broadening antenna, using the moment method 
and genetic algorithm to load the network number, position, and to load cell resistance to have optimized design, and the antenna test results show that the actual production of the antenna VSWR within $5 \sim 20 \mathrm{MHz}$ frequency range is less than 2.5, and the overall small size antenna is set up to facilitate the matching circuit that is very simple and suitable for arraying use.

\section{References}

[1] Gao Fei, Chen Yiling, Liu Qizhong, The Analysis of the Antenna[J], the Electronic Journal, 1999, 27(12): 124-125

[2]Gao Huotao, Yang Zijie, Li Jie, Wei Zhongwei, "The system shore-based high-frequency surface wave radar remote design of small broadband antenna” [J] Electronics \& Information Journal 20045: 1, 2-20

[3] Liu Chao, Liu Qizhong, Liang Yujun, Zhang Jianwei, "The research of ship-used shortwave broadband whip antenna” Radio Science Journal, 2006, 21(6): 955-958.

[4]Huang Youhuo, Liu Qizhong, Wei Wenbo, Ji Yicai, "Broadband matching networks POS optimized antenna design " [J], Microwave Journal, 2007, 5: 152-154.

[5] Liu Zhili, "The research of the matching network based on particle swarm optimization broadband antenna” Zhejiang: Harbin Engineering University, 2009. 